\title{
Caracterización del juego de azar en Galicia: un problema de Salud Pública
}

\section{Gambling characteristics in Galicia: a Public Health problem}

\author{
Julia Rey-Brandariz**, Mónica Pérez-Ríos**, Maria Isolina Santiago-Pérez*, \\ María lorenzo*, Alberto Malvar*, Xurxo Hervada*. \\ * Servicio de Epidemiología. Dirección General de Salud Pública, Santiago de Compostela. España. \\ ** CIBER de Epidemiología y Salud Pública, CIBERESP; Área de Medicina Preventiva y Salud Pública. Universidad da Santiago \\ de Compostela, Santiago de Compostela. España.
}

\section{Resumen}

Debido a la expansión de los juegos de azar, los trastornos asociados al juego se convierten en un gran problema social con una alta relevancia para la Salud Pública, afectando tanto a adultos como a adolescentes. Los principales objetivos de este estudio fueron conocer la prevalencia de gallegos que gastaron dinero en juegos de azar en el último año y de jugadores con un trastorno de juego o de riesgo. Los datos se obtuvieron del estudio transversal realizado en 2017 por el Sistema de Información sobre Conductas de Riesgo (SICRI). El SICRI se basa en la realización anual de encuestas telefónicas a la población gallega residente que tiene al menos 16 años de edad con la muestra equidistribuida en 12 meses. Un total de 7.841 participantes fueron seleccionados mediante un muestreo aleatorio estratificado. Con el objetivo de estimar la prevalencia de trastorno de juego o de riesgo se utilizó el cuestionario South Oaks Gambling Screen (SOGS) de Lesieur y Blume. Se estimó la prevalencia de juego y jugadores con un trastorno de juego o juego de riesgo y se ajustaron modelos de regresión para identificar las variables asociadas al trastorno de juego o juego de riesgo. De la población gallega de 16 años en adelante, el 58,1\% (IC 95\%: 57,0-59,2) gastó dinero en juegos de azar en los 12 meses previos a la realización del estudio, siendo la prevalencia de jugadores más alta en los hombres $(64,6 \%$ vs. $52,2 \%)$ en todos los grupos de edad. La prevalencia de trastorno de juego o juego de riesgo en la población es del 1,6\% (IC 95\%: 1,3-1,9), siendo más alta entre los hombres y en los jugadores más jóvenes. Las prevalencias obtenidas señalan al juego como un importante problema de Salud Pública, siendo los varones jóvenes los que tienen un mayor riesgo de desarrollar un problema asociado al juego.

Palabras clave: Juego de azar; Prevalencia; Adulto; Conducta Adictiva.

\begin{abstract}
Due to the increase in gambling, gambling disorders have become a major social problem of importance for public health, affecting both adults and adolescents. The main objectives of this study were to assess the prevalence of the Galician population who spent money on gambling in the last year and the prevalence of people with, or at risk of, gambling disorder. Data was obtained from a cross-sectional survey carried out in 2017 by the Galician Information System on Risk Behaviors (SICRI). The SICRI conducts annual telephone surveys of Galician residents who were at least 16 years of age, with the sample equidistributed over a 12-month period. A total of 7,841 participants were selected using stratified random sampling. In order to estimate the prevalence of gambling disorder or at-risk gambling, The South Oaks Gambling Screen (SOGS) questionnaire by Lesieur and Blume was applied. The prevalence of gambling and having or being at risk of gambling disorder was estimated and regression models were adjusted to identify variables associated with gambling disorder or being at risk. Of the Galician population aged 16 years and older, $58.1 \%$ (95\% CI: 57.0-59.2) spent money on gambling in the 12 months previous to this study, with the highest prevalence of gambling found in men $(64.6 \%$ vs. $52.2 \%)$ in all age groups. The prevalence of gambling disorder or at-risk gambling at the population level is $1.6 \%$ (95\% CI 1.3-1.9), and is higher among men and younger gamblers. The prevalence obtained signals to gambling as a major public health concern, with young males being at greater risk of developing a gambling problem. Keywords: Gambling; Prevalence; Adult; Behavior; Addictive.
\end{abstract}

Recibido: Agosto 2020; Aceptado: Diciembre 2020.

Enviar correspondencia a:

Mónica Pérez Ríos. CIBER de Epidemiología y Salud Pública, CIBERESP. Departamento de Medicina Preventiva y Salud Pública, Universidad de Santiago de Compostela, Santiago de Compostela, España. Telefono-Fax: 0034881812277.

E-mail: monica.perez.rios@usc.es. 


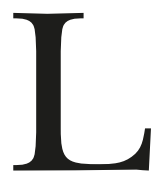

a práctica de juegos de azar es una actividad legal y socialmente aceptada. Datos recientes concluyen que la mayoría de los adultos han jugado a juegos de azar en algún momento de su vida y que a nivel poblacional hay más jugadores que no jugadores. La práctica de juegos de azar varía entre poblaciones y las prevalencias más altas se observan en países de Europa y en Estados Unidos. Así, en 2010 en el Reino Unido el 73,0\% de la población de 16 años y más declaró haber jugado en el último año; en 2011-2013 en Estados Unidos entre la población de 18 años en adelante este porcentaje se estimó en el 76,9\%, mientras que en Corea del Norte en el mismo grupo de edad en el año 2011 fue del 41,8\% (Calado y Griffiths, 2016). A pesar de que la mayoría de los adultos jugadores no desarrollan trastornos relacionados con el juego (Potenza, Kosten y Rounsaville, 2001), en otros ocasiona un trastorno adictivo que acarrea problemas de salud, sociales, laborales o económicos y altas tasas de suicidio (Nautiyal, Okuda, Hen y Blanco, 2017). En el mundo, la prevalencia de jugadores con problemas asociados al juego, referido al último año, varía entre el $0,1 \%$ y el $5,8 \%$; siendo Oceanía el continente en donde las prevalencias son más bajas y América del Norte en donde son más altas. Ser hombre, joven, tener un nivel de estudios bajo o pocos ingresos son variables que se asocian con los problemas de juego en diferentes estudios (Calado et al., 2016).

El desarrollo de las tecnologías de la comunicación e información (TIC) está ocasionando una revolución en los juegos de azar con la aparición de la modalidad de juego online, que aumenta considerablemente la accesibilidad a los juegos de azar en todo el mundo. Desde la publicación del Real Decreto de 1977 (Real Decreto-Ley 16/1977, de 25 de febrero, por el que se regulan los aspectos penales, administrativos y fiscales de los juegos de suerte, envite o azar y apuestas) no se realizaron cambios sustanciales en la legislación española hasta la Ley 13/2011 (Ley 13/2011, de 27 de mayo, de regulación del juego) en la que se legalizan los juegos online. Esta modalidad de juego es hoy en día una actividad económica en fase de crecimiento y se estima que en España, en términos económicos, supone una tercera parte del negocio del juego, así de los 41.826,8 millones de euros que se gastaron en juego en 2017, el $32,0 \%$ se gastó en juego online (Clotas, Bartroli, Caballé, Pasarín y Villalbí, 2020). En España los usuarios de juego online son mayoritariamente hombres $(83,3 \%)$ y menores de 46 años (87,5\%) (Dirección General de Ordenación del Juego, 2018). En los últimos años, debido a la expansión de los juegos de azar online, los trastornos del juego se convierten en un problema desde el punto de vista social y de alta relevancia para la Salud Pública (Abbott, 2020).

En España en 2015 el 75,7\% de la población de 18 años y más declaró haber gastado dinero en juegos de azar (Dirección General de Ordenación del Juego, 2015) y en 2017 este porcentaje fue del $60,2 \%$ entre la población de 15 a
64 años (Observatorio Español de las Drogas y las Adicciones y Delegación del Gobierno para el Plan Nacional sobre Drogas, 2019). En 1980 el juego patológico fue clasificado formalmente como un trastorno mental por la Asociación Americana de Psiquiatría (1980). En la última edición del Manual Diagnóstico y Estadístico de los Trastornos Mentales (DSM-V) (American Psychiatric Association, 2013), se recoge como un trastorno de naturaleza adictiva, incluyéndose dentro del capítulo de "Trastornos relacionados con sustancias y trastornos adictivos". El juego patológico es una conducta de juego persistente y desadaptativa que genera un malestar clínicamente significativo, (American Psychiatric Association, 2013), que afecta tanto a adultos como a adolescentes con una prevalencia en estos últimos en ocasiones superior a la de los adultos (Volberg, Gupta, Griffiths, Olason y Delfabbro, 2010). En 2015, el 0,3\% de la población española de 18 años en adelante tenía un trastorno de juego patológico (Dirección General de Ordenación del Juego, 2015) y en 2017 entre la población de 15 a 64 años este porcentaje se estimó en el 0,5\% (Observatorio Español de las Drogas y las Adicciones et al., 2019).

Debido a la necesidad de disponer de datos representativos para Galicia en relación con el juego, los objetivos de este estudio son: 1) conocer la prevalencia de gallegos que gastaron dinero en juegos de azar en el último año, 2) conocer que tipos de juegos son los más utilizados y la frecuencia de juego, 3) describir a los jugadores, 4) conocer la prevalencia de jugadores con un trastorno de juego o con un juego de riesgo; y 5) caracterizar a los jugadores con un trastorno de juego o con un juego de riesgo.

\section{Métodos}

El Sistema de Información sobre Conductas de Riesgo (SICRI) se implantó en el año 2005. Se basa en la realización de estudios transversales en los que se obtiene información con periodicidad anual entre la población de 16 años en adelante que reside en Galicia, a través de encuestas telefónicas empleando un sistema CATI (Computed-Assisted Telephone Interview).

La encuesta realizada en 2017 (SICRI-2017) se diseñó para garantizar la representatividad de la población definida en función del sexo y en cuatro grupos de edad: 16-24, 25-44, 45-64 y 65 años en adelante. Se realizó un muestreo aleatorio estratificado utilizando como marco de muestreo la base de datos de Tarjeta Sanitaria, que incluye tanto teléfonos fijos como móviles de todos los gallegos que tuvieron algún contacto con el sistema sanitario, y que tiene una cobertura de aproximadamente el $97,0 \%$ de la población residente en Galicia. El trabajo de campo fue llevado a cabo entre enero y diciembre de 2017 con la muestra equidistribuída por meses, realizándose una media de 650 encuestas al mes. 
En el cuestionario del SICRI-2017 se incluyeron preguntas específicas que permitían estimar la prevalencia de práctica de juegos de azar y de jugadores con trastornos de juego o con juego de riesgo. La práctica de juegos de azar se definió a partir de la respuesta afirmativa a la pregunta "Piense en los últimos 12 meses, ¿gastó usted dinero en juegos como la lotería, máquinas tragaperras, apuestas por internet...?”. A los que contestaban que sí, se les preguntaba en que tipos de juegos habían gastado dinero, estableciéndose 5 categorías de respuesta no excluyentes entre sí: Juegos de lotería (lotería, quiniela, primitiva, bonoloto, cupón); apuestas por internet; casinos/bingos; máquinas tragaperras y otros juegos. Además, para cada una de las opciones de respuesta, se les preguntaba por la frecuencia de práctica con 4 opciones de respuesta: todos los días, todas o casi todas las semanas, algunas veces al mes y algunas veces al año (Consellería de Sanidade, 2018).

Las personas que declararon haber jugado a juegos de azar en los 12 meses anteriores a la entrevista contestaron a 10 preguntas con el objetivo de estimar la prevalencia de jugadores con trastornos de juego o con juego de riesgo. Estas preguntas forman parte del bloque de dependencia al juego del cuestionario South Oaks Gambling Screen (SOGS) de Lesieur y Blume (1987) adaptado al español (Echeburúa, Martín-Baez, Fernández-Montalvo y Páez, 1994). Las preguntas pertenecientes al bloque del endeudamiento no se incluyeron. La puntuación total del cuestionario varía de 0 a 10 puntos. Para la identificación de un jugador con un problema de adicción al juego se mantiene el punto de corte propuesto en la validación del cuestionario SOGS en España; así, se clasifican como jugadores con un trastorno de juego a aquellos que obtienen 4 puntos o más y como jugadores con un juego de riesgo a aquellos que obtienen 2 o 3 puntos.

A partir de la información obtenida se calcularon prevalencias en global y en función del sexo y grupo de edad (16-24, 25-44, 45-64 y 65 años en adelante): porcentaje de personas que gastaron dinero en juegos de azar en el último año, en global y para cada tipo de juego; porcentaje de jugadores que gastaron dinero en la lotería todas las semanas, algunas veces al mes y algunas veces al año y la prevalencia de jugadores con un trastorno de juego o con un juego de riesgo, que son aquellos que tienen 2 o más puntos en el SOGS.

Para caracterizar a los jugadores con un trastorno de juego o con un juego de riesgo se ajustó un modelo de regresión logística incluyendo inicialmente todas las variables que resultaron significativas en un análisis bivariante previo, con $\mathrm{p}<0,1$; en el modelo final se mantuvieron las variables con $\mathrm{p}<0,05$. A partir de ese modelo se calcularon odds ratios (OR) ajustadas. Se valoraron variables sociodemográficas: sexo; grupo de edad: 16-24, 25-44, 45-64 y 65 y más años; país de nacimiento: España-otro país; ámbito de residencia: rural, semiurbano y urbano; situación lab- oral en el momento de la encuesta: trabaja, en paro o sin trabajo, labores del hogar, pensionista, estudiante $u$ otra situación; nivel de estudios en tres categorías: básico (educación primaria o menos), medio (educación secundaria) y superior (universitarios); y convivencia en pareja: si-no. Además, se valoró la autopercepción del estado de salud en el momento de la encuesta: muy bueno, bueno, normal, malo y muy malo. Se analizaron variables relacionadas con la conducta: consumo de tabaco y cannabis categorizadas ambas como fumador o no fumador en el momento de la encuesta; consumo de alcohol a través del cuestionario AUDIT considerándose como indicativo de consumo de riesgo o problemático una puntuación $\geq 8$ (7 en las mujeres y en los mayores de 64 años); uso de aplicaciones de mensajería instantánea, redes sociales y juegos de realidad virtual durante más de dos horas diarias, jugar a juegos de azar diferentes a la lotería, jugar a la lotería todas las semanas o el uso problemático de internet (UPI), definido a partir de una puntuación de $\geq 25$ en el Cuestionario de Experiencias Relacionadas con Internet (CERI). Las prevalencias y los OR ajustados se presentan con intervalos de confianza del 95\% (IC95\%). El análisis se realizó con la muestra ponderada de acuerdo al diseño muestral, y para los cálculos se utilizó Stata v14.2.

Este estudio se realizó siguiendo las normas de buena práctica y la Declaración de Helsinki, de forma que se solicitó y se obtuvo consentimiento expreso verbal de la persona encuestada.

\section{Resultados}

En el SICRI-2017 se entrevistaron 7.841 personas mayores de 15 años, la tasa de respuesta fue del $78 \%$.

E1 58,1\% (IC95\%: 57,0-59,2) de la población gallega de 16 años y más gastó dinero en juegos de azar en los 12 meses previos a la realización del estudio. La prevalencia de jugadores aumentó a medida que avanza la edad, alcanzando el máximo en el grupo de 45-64 años, tanto en hombres $(73,1 \%)$ como en mujeres $(62,8 \%)$, disminuyendo a continuación (Figura 1). La prevalencia de jugadores es más alta en los hombres, tanto en global (64,6\% vs. 52,2\%), como por grupo de edad, si bien las diferencias se acentúan en los grupos de edad extremos (16-24 y $\geq 75)$ en los cuales las diferencias superan los 20 puntos porcentuales (Figura 1).

Teniendo en cuenta el tipo de juego en el que gastaron el dinero los jugadores gallegos en los últimos 12 meses, el 97,9\% (IC 95\%: 97,5-98,2) declaró haber gastado en lotería, quinielas, primitiva, bonoloto y juegos similares; seguido de las apuestas a través de internet con una prevalencia del 2,1\% (IC 95\%: 1,7-2,4) (Tabla 1).

$\mathrm{Al}$ valorar la frecuencia de juego entre los gallegos que declararon jugar, la periodicidad anual es la más frecuente, con independencia del sexo y el grupo de edad. Entre 


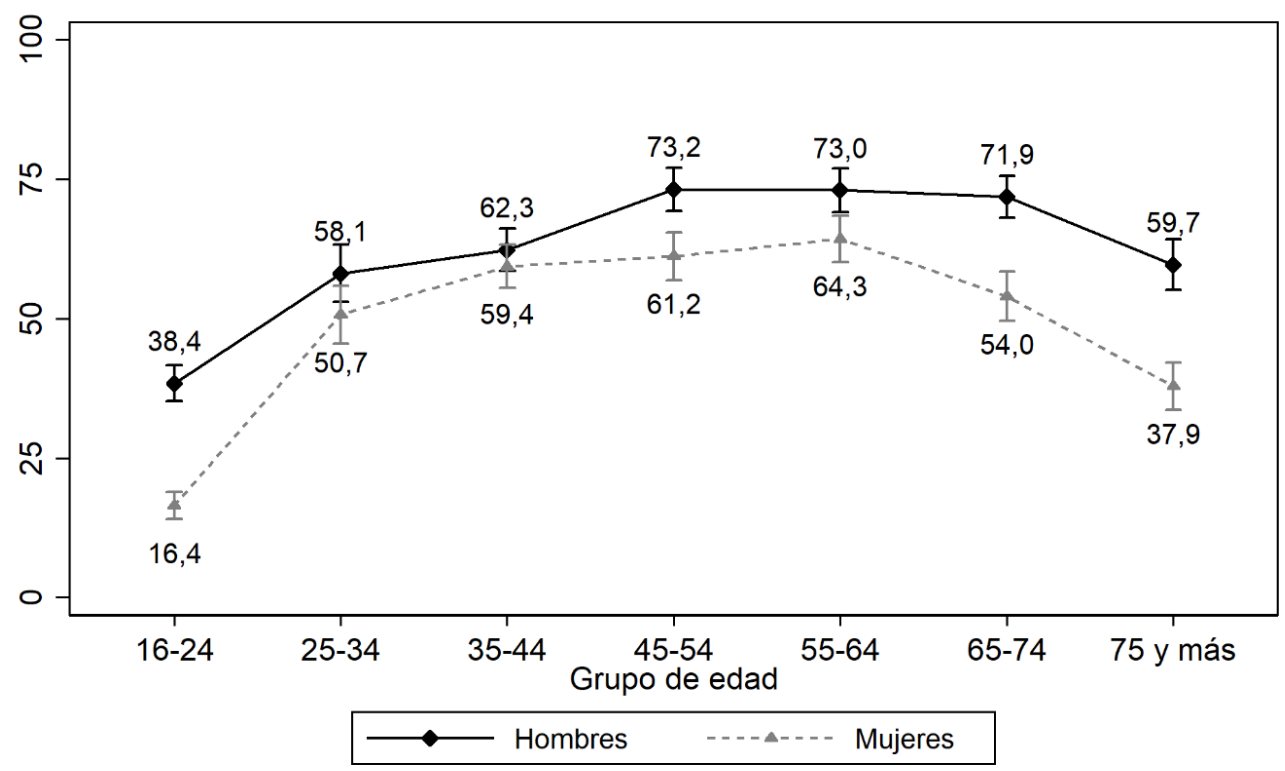

Figura 1. Porcentaje de población de 16 años en adelante que gastó dinero en juegos en los últimos 12 meses, en función del sexo y el grupo de edad, con un intervalo de confianza del 95\% (IC95\%). Galicia, SICRI-2017.

Tabla 1. Porcentaje de personas de 16 años en adelante que gastaron dinero en juegos de azar en los últimos 12 meses en función del tipo de juego. Datos en toda la población, en los jugadores y en los jugadores con trastorno de juego o juego de riesgo, con un intervalo de confianza del 95\% (IC95\%). Galicia, SICRI-2017.

\begin{tabular}{|c|c|c|c|c|c|c|}
\hline \multirow[t]{2}{*}{ Tipo de juego } & \multicolumn{2}{|c|}{ En toda la población } & \multicolumn{2}{|c|}{ En los jugadores } & \multicolumn{2}{|c|}{$\begin{array}{c}\text { En los jugadores con trastorno de } \\
\text { juego o juego de riesgo }\end{array}$} \\
\hline & $\%$ & IC95\% & $\%$ & IC95\% & $\%$ & IC95\% \\
\hline Lotería, quinielas, primitiva & 56,9 & $55,7-58,0$ & 97,9 & $97,5-98,2$ & 88,9 & $84,2-93,6$ \\
\hline Otros juegos & 2,1 & $1,8-2,3$ & 3,5 & $3,1-4,0$ & 24,3 & $17,1-31,5$ \\
\hline Apuestas por internet & 1,2 & $1,0-1,4$ & 2,1 & $1,7-2,4$ & 14,1 & $8,6-19,5$ \\
\hline Casino/Bingo & 0,3 & $0,2-0,3$ & 0,4 & $0,3-0,6$ & 2,4 & $0,4-4,3$ \\
\hline Máquinas tragaperras & 0,5 & $0,3-0,6$ & 0,8 & $0,6-1,1$ & 10,2 & $4,8-15,5$ \\
\hline Apuestas deportivas & 0,3 & $0,2-0,4$ & 0,5 & $0,4-0,7$ & 1,9 & $0,5-3,3$ \\
\hline
\end{tabular}

la población gallega que declara jugar a lotería, quinielas o primitiva, el 33,9\% (IC 95\%: 32,4-35,4) lo hacen con periodicidad semanal, siendo la prevalencia más alta en los hombres (41,8\% vs. 25,2\%). En función del grupo de edad, la frecuencia del juego semanal más baja se observa entre los jugadores de edades comprendidas entre los 16 y 24 años [16,2\% (IC 95\%: 12,3-20,2)] y la más alta entre los de 45 a 64 años [37,9\% (IC 95\%: 35,3-40,4)] (Figura 2).

El 1,2\% (IC95\%: 1,0-1,4) de los gallegos de 16 años en adelante gastaron dinero en apuestas online en el último año. La prevalencia varía tanto por sexo como por grupo de edad, siendo más alta en los hombres $(2,4 \%$ vs. $0,1 \%)$ y disminuyendo con la edad (Tabla 2). La prevalencia de apuestas online alcanzó el 6,3\% (IC 95\%: 5,2-7,4) entre los gallegos de 16 a 24 años frente al 0,2\% (IC 95\%: 0,1-0,3) en la población de 45 años en adelante. Entre los jugadores, el 2,1\% (IC 95\%: 1,7-2,4) declaró hacer apuestas online, alcanzando la prevalencia un valor máximo del 22,8\% (IC 95\%: 19,2-26,5) en los jugadores más jóvenes.
En relación con la adicción al juego, el 90,2\% de los gallegos que declararon haber jugado a algún juego de azar en el último año no obtuvo ningún punto en el cuestionario SOGS y el $7 \%$ obtuvo 1 punto (Tabla 3). La prevalencia poblacional de trastorno de juego o de juego de riesgo es del 1,6\% (IC 95\%: 1,3-1,9); clasificándose un 0,4\% (IC 95\%: 0,3-0,6) como trastorno de juego y un 1,2\% (IC 95\%: 1,0-1,5) como juego de riesgo. Restringiendo este análisis a los jugadores, esta prevalencia es del 2,8\% (IC 95\%: 2,33,3): $0,7 \%$ (IC 95\%: 0,5-1,0) de jugadores con un trastorno de juego y el 2,1\% (IC 95\%: 1,6-2,5) con un juego de riesgo (Figura 3). La prevalencia de trastorno de juego entre los jugadores es más alta entre los hombres $(1,3 \%$ vs. $0,1 \%)$ y entre los 16-24 años (2,4\%) (Figura 3). En cuanto al tipo de juego, los jugadores con trastorno de juego o juego de riesgo juegan mayoritariamente a lotería, quinielas, primitiva, bonoloto y juegos similares, al igual que el conjunto de jugadores, pero el porcentaje en éstos últimos es menor [88,8\% (IC 95\%: 84,2-93,6) ]; en cambio, es mayor en las 


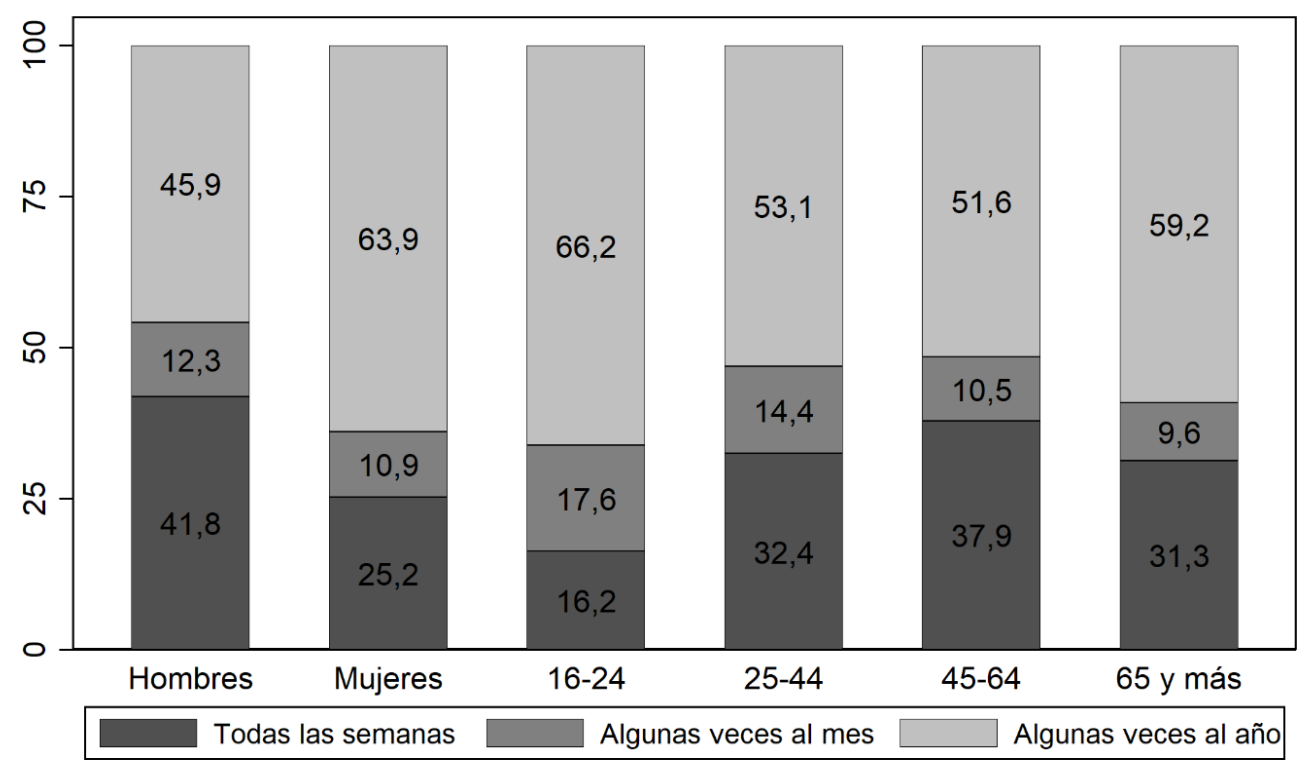

Figura 2. Distribución porcentual de los jugadores de loterías, quinielas o primitiva en función de la frecuencia del juego. Los datos se presentan en función del sexo y del grupo de edad. Galicia, SICRI-2017.

Tabla 2. Porcentaje de personas de 16 años en adelante que gastaron dinero en apuestas por internet en los últimos 12 meses, en función del sexo y grupo de edad. Datos en toda la población y en los jugadores, con un intervalo de confianza del 95\% (IC95\%). Galicia, SICRI-2017.

\begin{tabular}{|c|c|c|c|c|c|c|c|c|}
\hline \multirow[b]{3}{*}{ Todos } & \multicolumn{4}{|c|}{ En toda la población } & \multicolumn{4}{|c|}{ En los jugadores } \\
\hline & \multirow{2}{*}{$\begin{array}{c}\mathrm{n} \\
7.841\end{array}$} & \multirow{2}{*}{$\begin{array}{c}\% \\
1,2\end{array}$} & \multicolumn{2}{|c|}{ IC95\% } & \multirow{2}{*}{$\begin{array}{c}n \\
4.142\end{array}$} & \multirow{2}{*}{$\begin{array}{c}\% \\
2,1\end{array}$} & \multicolumn{2}{|c|}{ IC95\% } \\
\hline & & & 1,0 & 1,4 & & & 1,7 & 2,4 \\
\hline \multicolumn{9}{|l|}{ Por sexo } \\
\hline Hombres & 3.914 & 2,4 & 1,9 & 2,8 & 2.346 & 3,7 & 3,0 & 4,4 \\
\hline Mujeres & 3.927 & 0,1 & 0,0 & 0,2 & 1.796 & 0,2 & 0,1 & 0,4 \\
\hline \multicolumn{9}{|l|}{ Por grupo de edad } \\
\hline 16-24 años & 1.829 & 6,3 & 5,2 & 7,4 & 501 & 22,8 & 19,2 & 26,5 \\
\hline 25-44 años & 2.007 & 2,0 & 1,4 & 2,6 & 1.159 & 3,3 & 2,3 & 4,4 \\
\hline 45 años y más & 4.005 & 0,2 & 0,1 & 0,3 & 2.482 & 0,3 & 0,1 & 0,5 \\
\hline
\end{tabular}

Tabla 3. Distribución de la población y de los jugadores de 16 años en adelante en función de la puntuación obtenida en el cuestionario South Oaks Gambling Screening. Porcentajes con intervalos de confianza del $95 \%$ (IC95\%). Galicia, SICRI-2017.

\begin{tabular}{lcccccc}
\hline & \multicolumn{3}{c}{ En toda la población } & \multicolumn{3}{c}{ En los jugadores } \\
\cline { 2 - 7 } & $\%$ & \multicolumn{2}{c}{ IC95\% } & $\%$ & \multicolumn{2}{c}{ IC95\% } \\
\hline No juega & 41,9 & 40,8 & 43,1 & & & \\
0 puntos & 52,4 & 51,2 & 53,5 & 90,2 & 89,3 & 91,1 \\
1 punto & 4,1 & 3,6 & 4,5 & 7,0 & 6,2 & 7,8 \\
2 puntos & 0,9 & 0,7 & 1,1 & 1,6 & 1,2 & 2,0 \\
3 puntos & 0,3 & 0,2 & 0,4 & 0,5 & 0,3 & 0,7 \\
>=4 puntos & 0,4 & 0,3 & 0,6 & 0,7 & 0,5 & 1,0 \\
\hline
\end{tabular}

apuestas por internet $[14,1 \%$ (IC 95\%: 8,6-19,6) ] y máquinas tragaperras [10,2\% (IC 95\%: 4,5-15,5)] (Tabla 1).

Todas las variables relacionadas con la conducta se asociaron significativamente con el trastorno de juego o juego de riesgo en el análisis bivariante $(\mathrm{p}<0,05)$; entre las variables sociodemográficas, la asociación fue significativa para el sexo, el grupo de edad, el nivel de estudios y la convivencia en pareja. Al ajustar el modelo multivariante, se encontró que las características asociadas de forma independiente con el trastorno de juego o el juego de riesgo son ser varón, tener un consumo de alcohol de riesgo, pasar más de 2 horas diarias usando aplicaciones de mensajería instantánea, tener un uso problemático de internet, jugar a otro tipo de juegos de azar diferentes a la lotería y jugar a la lotería todas las semanas. El OR ajustado de cada variable, junto con su intervalo de confianza del $95 \%$, se mues- 

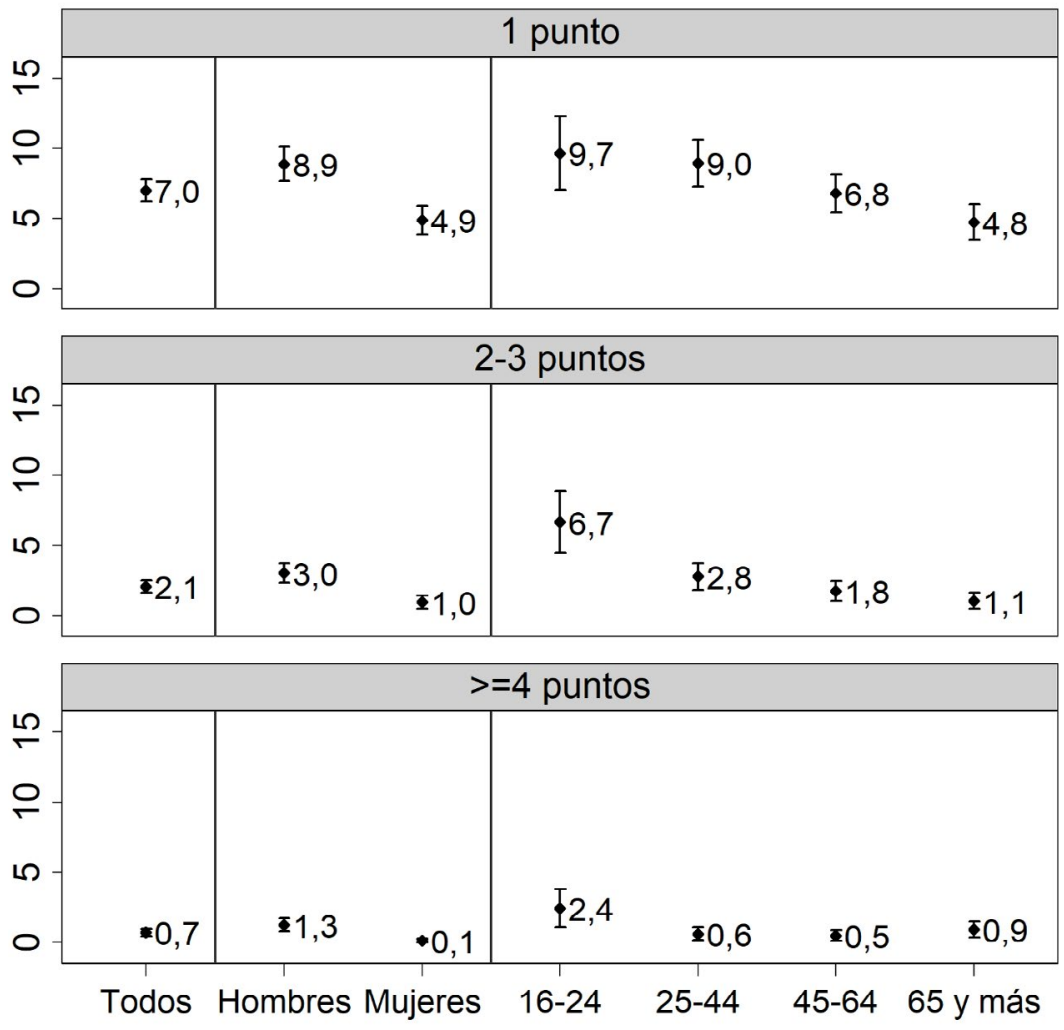

Figura 3. Porcentaje de jugadores de 16 años en adelante que obtuvieron 1 punto, 2-3 puntos y 4 puntos o más en el cuestionario South Oaks Gambling Screening, por sexo y grupo de edad, con un intervalo de confianza del 95\% (IC95\%). Galicia, SICRI-2017.

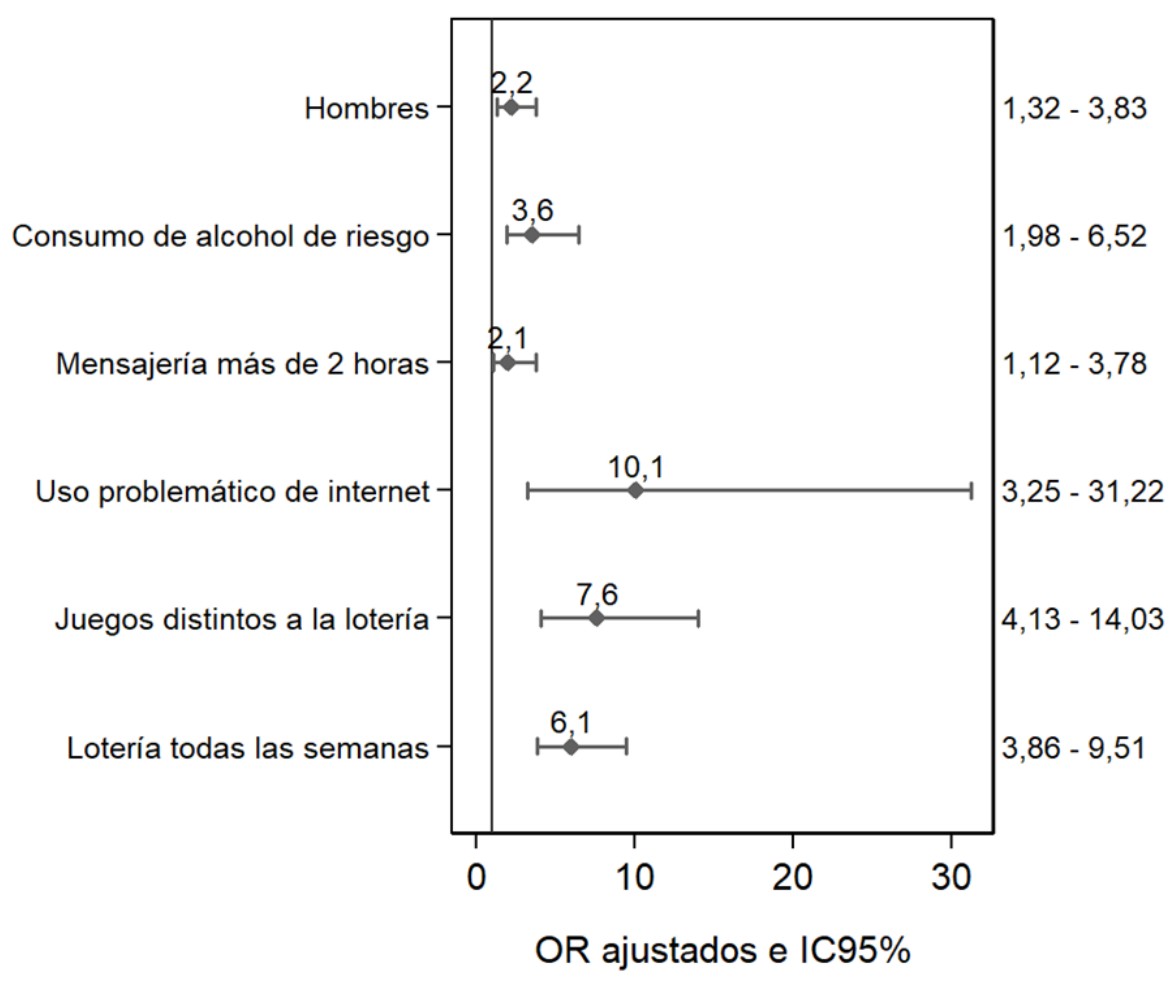

Figura 4. Características asociadas al trastorno de juego o el riesgo de tenerlo. Odds ratios (OR) ajustados con un intervalo de confianza del 95\% (IC95\%). Galicia, SICRI-2017.

tra en la Figura 4, donde se observa que todos los valores son superiores a 2, y que el uso problemático de internet multiplica por 10 la probabilidad de tener un trastorno del juego o juego de riesgo. 


\section{Discusión}

En el último año, el 58,1\% de la población gallega mayor de 15 años gastó dinero en juegos de azar, principalmente en lotería, quinielas, primitiva y similares. La prevalencia de jugadores es más alta en hombres y en la población de 45 a 64 años. Entre los gallegos de 16 a 24 años, 16 de cada 100 realiza apuestas online. El 0,4\% de los gallegos tienen un trastorno de juego y el 1,2\% un juego de riesgo; entre los jugadores estas prevalencias son del $0,7 \%$ y del 2,1\%, respectivamente.

La prevalencia de jugadores estimada para Galicia en este estudio es inferior a la obtenida para el conjunto de España en el estudio sobre prevalencia de juegos de azar en población de 15 años y más realizado en 2015(67,0\%) (Dirección General de Ordenación del Juego, 2015), y similar a la estimada por la Encuesta sobre alcohol y otras drogas en España (EDADES) en 2017 entre la población de 15 a 64 años (60,2\%) (Observatorio Español de las Drogas y las Adicciones et al., 2019). Las características sociodemográficas de los jugadores identificadas en ambos estudios son similares, siendo el perfil del jugador ser hombre con edades comprendidas entre los 45 y 64 años.

En Galicia, igual que en EDADES-2017, entre las modalidades de juego más frecuentes destacan la lotería, quiniela, primitiva o bonoloto; si bien las apuestas online fueron la segunda opción. Así, 1 de cada 100 gallegos de 16 años en adelante, 25.890, hicieron apuestas por internet y de estos 10.278 tenían edades comprendidas entre los 16 y 24 años. Este aspecto es importante ya que precisamente los juegos de azar online son más adictivos que cualquier otro tipo de juego (Monaghan, 2009; Wood, Williams y Parke, 2012) y son la principal causa de adicción al juego en la gente más joven (Chóliz, 2016). El 16,2\% de los estudiantes europeos de 16 años reconocieron haber apostado online en los últimos 12 meses (Molinaro et al., 2018). En Galicia, en este estudio, estimamos la prevalencia de apuestas online en los jóvenes de 16 años en el 11,9\% (dato no mostrado en resultados). De hecho, aunque el juego en menores sea una actividad ilegal, según datos del SICRI-2017 (no mostrados en resultados) hasta un $19,4 \%$ de los varones y un $3,8 \%$ de las mujeres entre los 16 y 17 años gastaron dinero en algún tipo de juego de azar, ya fuese presencial o de modalidad online. Cifras superiores fueron obtenidas entre estudiantes de 16 años en Europa (31,6\% en hombres vs. 13,8\% mujeres) (Molinaro et al., 2018). Desde el punto de vista clínico estos datos son preocupantes, ya que la exposición temprana al juego aumenta el riesgo de problemas relacionados con el mismo en la vida adulta (Burge, Pietrzak, Molina y Petry, 2004; Dowling et al., 2017).

Aunque podría parecer que en Galicia la prevalencia de problemas asociados al juego es baja, el 0,4\% de los gallegos son jugadores con un trastorno de juego y el 1,2\% con un juego de riesgo. Las prevalencias obtenidas apuntan a que 38.399 gallegos tienen problemas con el juego. Ade- más, es importante reseñar que es precisamente entre la población más joven, de 16 a 24 años, donde se observa la mayor prevalencia, $9,1 \%$.

En Galicia se realizaron 3 estudios previos, en los años 1991, 1993 y 2001, que estimaron la prevalencia de problemas relacionados con el juego en la población adulta (Becoña, 1993; Becoña y Fuentes, 1995; Becoña, 2004). Cambios en el diseño, en el instrumento de medición o en la edad de la población a estudio no permiten valorar de forma precisa la evolución de la prevalencia de problemas con el juego entre la población gallega. Desde las primeras estimaciones hasta las actuales se observa un descenso en los estimadores puntuales de las prevalencias, pero la presentación de los resultados en los estudios previos, que no incluyen la precisión en las estimaciones presentadas (en ningún caso se incluyen intervalos de confianza), podría colocarnos en un escenario de estabilización de las prevalencias. Así, el estudio realizado en Galicia en 1993, y que empleó el SOGS, estimó la prevalencia de jugadores con un trastorno del juego (juego patológico en el estudio) en el $1,4 \%(n=14)$ y de jugadores con un juego problemático (jugadores problema en el estudio) en el 2,0\% ( $n=21)$ (Becoña et al., 1995); en este estudio nosotros hemos estimado estas prevalencias con el mismo instrumento en el $0,7 \%(0,5-1,0)$ y en el $2,1 \%(1,6-2,5)$, respectivamente, por lo que los cambios observados en los estimadores puntuales de la prevalencia de jugadores con trastorno del juego podrían ser residuales. Las últimas estimaciones disponibles para Galicia son de un estudio realizado en 2001 en población de 18 años y más aplicando el NODS (National Opinion Research Center DSM-IV Screen for Gambling Problems). En este estudio se estimó que la prevalencia de jugadores en riesgo era del $0,25 \%$, jugadores con un juego problemático $0,25 \%$ y trastorno de juego $0,31 \%$ (Becoña, 2004). Estas prevalencias son inferiores a las que hemos obtenido para Galicia en 2017 y a las que se obtuvieron en España en 2015 aplicando el cuestionario NODS (Dirección General de Ordenación del Juego, 2015). Si bien se debe tener en cuenta que el NODS es mucho más restrictivo a la hora de identificar a personas con problemas de juego en comparación con el SOGS, que según algunos autores podría sobreestimar las prevalencias cuando se aplica a muestras poblacionales (Raylu y Oei, 2002). Entre ambos estudios han transcurrido 16 años y el juego ha cambiado en nuestro entorno (Abbott, 2020).

Las características de los gallegos con trastornos de juego o con un juego de riesgo coinciden con las de otros estudios realizados en España; aumentando la probabilidad entre los hombres y entre aquellos jugadores con un consumo de alcohol de riesgo (Echeburúa, González-Ortega, de Corral y Polo-López, 2013; Jauregui, Estévez y Urbiola, 2016). El uso problemático de internet o de mensajería más de 2 horas al día son otras de las características que aumentan la probabilidad de trastornos de juego o de jue- 
go de riesgo. Estudios previos ya habían valorado que las personas con estos comportamientos compartían rasgos similares de personalidad y de distress emocional (Mallorquí-Bagué et al., 2017).

Entre las limitaciones de este estudio cabe destacar que para estimar la prevalencia de trastorno de juego o de juego de riesgo no se aplicaron las preguntas incluidas en el cuestionario SOGS relacionadas con el endeudamiento. Esto podría causar una infraestimación de la prevalencia, ya que no se incluyen las 9 preguntas sobre a quién se le pide el dinero para gastar en juego. Es importante explicar que las preguntas de endeudamiento están dirigidas a aquellas personas que contestan de forma positiva a la pregunta: “¿En alguna ocasión pidió dinero prestado para jugar o para pagar deudas del juego?”. De las 4.142 personas que afirmaron haber gastado dinero en el juego en los 12 meses previos a la realización de la encuesta, solamente 19 contestaron de forma afirmativa a esta pregunta y de estos, 12 ya tenían 4 puntos o más en las preguntas previas, por lo cual ya estaban clasificados como jugadores con un trastorno de juego. Dos de ellos contaban con 3 puntos, y dado que tendrían un punto más por lo menos en las preguntas de endeudamiento, pasaron a clasificarse como jugadores con un trastorno de juego. Solamente 5 casos que tenían 1 o 2 puntos fueron excluidos del análisis ya que no se podía saber si en las preguntas de endeudamiento sumarían uno o más puntos. Esto nos permite concluir que el impacto que pueden tener estas preguntas relacionadas con el endeudamiento en la estimación de la prevalencia de trastorno de juego es muy bajo. Por lo cual, la no inclusión de estas preguntas, que consumen mucho tiempo en la aplicación del cuestionario, en este caso tienen un impacto imperceptible en la prevalencia estimada. Por otro lado, al haber identificado solo 37 personas con trastorno de juego, no ha sido posible un análisis comparativo entre sus características y las del resto de la muestra.

Entre los sesgos que podrían afectar a los resultados de este estudio destaca la deseabilidad social, que se puede manifestar con la ocultación de la práctica del juego, y que afectaría especialmente a los jugadores con un trastorno de juego. La inclusión de las preguntas de juego dentro de un cuestionario que valora otras conductas, tanto de riesgo como protectoras, podría mitigar el impacto de este sesgo. Entre las fortalezas de este estudio cabe destacar el elevado tamaño muestral y una tasa de respuesta próxima al 80,0\%; el primero garantiza una buena precisión de los resultados, y ambas aseguran la representatividad de la muestra.

Como conclusión, el porcentaje de población que gasta dinero en juegos de azar es inferior en Galicia que, en España en su conjunto, pero aun así las prevalencias obtenidas señalan al juego como un relevante problema de Salud Pública. La prevalencia de jugadores, tanto hombres como mujeres, alcanza el máximo en el grupo de 45 a 64 años, pero son los varones jóvenes los que tienen una ma- yor prevalencia de problemas asociados al juego por lo que las medidas de prevención tanto primarias, secundarias o terciarias deben estar orientas a ellos, sin olvidar a otros grupos vulnerables. La administración autonómica gallega, sensibilizada con el problema que se señala, trabaja en la modificación de la Ley 14/1985 Reguladora de los Juegos y Apuestas en Galicia. Esta revisión de la ley, al igual que ya se hizo con la regulación de otras áreas de salud que tenían que ver con el cambio de conductas y con la adopción de estilos de vida saludables como el consumo de tabaco, incorpora medidas tanto educativas como regulatorias que protejan a la población, especialmente a los colectivos más vulnerables, y que permitan reducir la prevalencia de trastornos de juego.

\section{Conflicto de intereses}

Todos los autores declaran no tener conflictos de intereses.

\section{Referencias}

Abbott, M. W. (2020). The changing epidemiology of gambling disorder and gambling-related harm: public health implications. Public Health, 184, 41-45. doi:10.1016/j. puhe.2020.04.003.

American Psychiatric Association (1980). Diagnostic and Statistical Manual of Mental Disorders (3rd ed).

American Psychiatric Association (2013). Diagnostic and Statistical Manual of Mental Disorders (5th ed).

Becoña, E. (1993). The prevalence of pathological gambling in Galicia (Spain). Journal of Gambling Studies, 9, 353-369. doi:10.1007/BF01014627.

Becoña, E. (2004). Prevalencia del juego patológico en Galicia mediante el NODS. ¿Descenso de la prevalencia o mejor evaluación del trastorno? Adicciones, 16, 173-184. doi:10.20882/adicciones.399.

Becoña, E. y Fuentes, M. J. (1995). El juego patológico en Galicia evaluado por el South Oaks Gambling Screen. Adicciones, 7, 423-440.

Burge, A. N., Pietrzak, R. H., Molina, C. A. y Petry, N. M. (2004). Age of gambling initiation and severity of gambling and health problems among older adult problem gamblers. Psychiatric Services, 55, 1437-1439. doi:10.1176/appi.ps.55.12.1437.

Calado, F. y Griffiths, M. D. (2016). Problem gambling worldwide: An update and systematic review of empirical research (2000-2015). Journal of Behavioral Addictions, 5, 592-613. doi:10.1556/2006.5.2016.073.

Clotas, C., Bartroli, M., Caballé, M., Pasarín, M. I. y Villalbí, J. R. (2020). El negocio de los juegos de azar: una perspectiva desde la salud pública. Revista Española de Salud Pública, 94, e202006043. 
Chóliz M. (2016). The challenge of online gambling: The effect of legalization on the increase in online gambling addiction. Journal of Gambling Studies, 32, 749-756. doi:10.1007/s10899-015-9558-6.

Consellería de Sanidade. (2018). SICRI-2017. Sevizo Galego de Saúde. Recuperado de https://www.sergas.gal/ Saude-publica/SICRI-2017?idioma=es.

Dirección General de Ordenación del Juego (2015). Estudio sobre prevalencia, comportamiento y características de los usuarios de juego de azar en España. Dirección General de Ordenación del Juego. Madrid: Ministerio de Hacienda y Administraciones Públicas. Recuperado de https:// www.ordenacionjuego.es/es/estudio-prevalencia.

Dirección General de Ordenación del Juego. (2018). Informe del Jugador Online. Dirección General de Ordenación del Juego. Madrid: Ministerio de Hacienda y Administraciones Públicas. Recuperado de https:/ / www.ordenacionjuego.es/es/informe-jugador-online.

Dowling, N. A., Merkouris, S. S., Greenwood, C. J., Oldenhof, E., Toumbourou, J. W. y Youssef, G. J. (2017). Early risk and protective factors for problem gambling: A systematic review and meta-analysis of longitudinal studies. Clinical Psychology Review, 51, 109-124. doi:10.1016/j. cpr.2016.10.008.

Echeburúa, E., Martín-Baez, C., Fernández-Montalvo, J. y Páez, D. (1994). Cuestionario de juego patológico de South Oaks (SOGS): Validación española. Análisis y Modificación de la Conducta, 20, 769-791.

Echeburúa, E., González-Ortega, I., De Corral, P. y Polo-López, R. (2013). Pathological gamblers and a non-psychiatric control group taking gender differences into account. The Spanish Journal of Psychology, 16, E2. doi:10.1017/sjp.2013.2.

Jauregui, P., Estévez, A. y Urbiola, I. (2016). Pathological Gambling and Associated Drug and Alcohol Abuse, Emotion Regulation, and Anxious-Depressive Symptomatology. Journal of Behavioral Addictions, 5, 251-260. doi:10.1556/2006.5.2016.038.

Lesieur, H. R. y Blume, S. B. (1987). The South Oaks Gambling Screen (SOGS): a new instrument for the identification of pathological gamblers. American Journal of Psychiatry, 144, 1184-1188. doi:10.1176/ajp.144.9.1184.

Ley 13/2011, de 27 de mayo, de regulación del juego. Boletín Oficial del Estado, 127, de 28 de mayo de 2013. Recuperado de https://www.boe.es/eli/es/l/2011/05/27/13/ con.

Mallorquí-Bagué, N., Fernández-Aranda, F., Lozano-Madrid, M., Granero, R., Mestre-Bach, G., Baño, M.,... Jiménez-Murcia, S. (2017). Internet gaming disorder and online gambling disorder: Clinical and personality correlates. Journal of Behavioral Addictions, 6, 669-677. doi:10.1556/2006.6.2017.078.

Molinaro, S., Benedetti, E., Scalese, M., Bastiani, L., Fortunato, L., Cerrai, S., ... Urdih Lazar, T. (2018). Prevalence of youth gambling and potencial influence of substance use and other risk factors throughout 33 European countries: first results from the 2015 ESPAD study. Addiction, 113, 1862-1873. doi:10.1111/add.14275.

Monaghan, S. M. (2009). Internet gambling - not just a fad. International Gambling Studies, 9, 1-4. doi: 10.1080/14459790902793077.

Nautiyal, K. M., Okuda, M., Hen, R. y Blanco, C. (2017). Gambling disorder: an integrative review of animal and human studies. Annals of the New York Academy of Sciences, 1394, 106-127. doi:10.1111/nyas.13356.

Observatorio Español de las Drogas y las Adicciones y Delegación del Gobierno para el Plan Nacional sobre Drogas. (2019). Adicciones comportamentales. Juego con dinero, uso de videojuegos y uso compulsivo de internet en las encuestas de drogas y otras adicciones en España EDADES y ESTUDES. Recuperado de https://pnsd.sanidad.gob. es/eu/profesionales/sistemasInformacion/sistemaInformacion/pdf/2019_Informe_adicciones_comportamentales_2.pdf.

Potenza, M. N., Kosten, T. R. y Rounsaville, B. J. (2001). Pathological gambling. JAMA, 286, 141-144. doi:10.1001/ jama.286.2.141.

Raylu, N. y Oei, T. P. (2002). Pathological gambling: A comprehensive review. Clinical Psychology Review, 22, 10091061. doi:10.1016/S0272-7358(02)00101-0.

Real Decreto-Ley 16/1977, de 25 de febrero, por el que se regulan los aspectos penales, administrativos y fiscales de los juegos de suerte, envite o azar y apuestas. Boletin Oficial del Estado, 56, de 7 de julio de 1977, 5302 a 5304. Recuperado de https://www.boe.es/eli/es/ $\mathrm{rdl} / 1977 / 02 / 25 / 16$.

Volberg, R. A., Gupta, R., Griffiths, M. D., Olason, D. T. y Delfabbro, P. (2010). An international perspective on youth gambling prevalence studies. International Journal of Adolescent Medicine and Health, 22, 3-38.

Wood, R. T., Williams, R. J. y Parke, J. (2012). The relationship between Internet gambling and problem gambling . En: R. J. Williams, R. T. Wood y J. Parke (Eds.), Routledge International Handbook of Internet Gambling (pp. 200-211). London: Routledge. 
